\title{
Challenges to conducting a 12-month longitudinal qualitative study on Chinese primary care physicians

\author{
Man Kay Poon ${ }^{1 *}$, Tai Pong Lam ${ }^{1 \#}$ \\ ${ }^{1}$ Department of Family Medicine and Primary Care
}

Li Ka Shing Faculty of Medicine, The University of Hong Kong, Pokfulam Road, Hong Kong

*elomkpoon@yahoo.com

\#tplam@hku.hk

\begin{abstract}
Background: Qualitative approach is commonly adopted in medical education research. However, it is difficult to conduct research studies on primary care physicians (PCPs). Conducting longitudinal qualitative studies is even more challenging. No studies on Chinese PCPs in this area are identified. Methods: We invited 14 Chinese PCPsto participate in a 12-month longitudinal qualitative studywhich explored their continuing professional learning experiences and viewpoints. They reported their learning activities byquestionnaires in which the findings served as a guide for subsequent interviews. Each of them took part in four individual interviewsspanning 12 months. We detailed the conduction process and challenges in a researchdiary and analyzed these data by thematic content analysis. Results: The major challenges were identification and recruitment of eligible PCPs. We tackled these challenges with the assistance of our professional networks and opinion leaders. We offered the participants an intellectual incentive by designing our study relevant to their profession. Flexibility and convenience of data collection could minimize the heavy burden on them. Conclusions:With appropriate recruitment strategies and planning of the research methods, researchers can successfully conduct longitudinal qualitative studies on Chinese PCPs about their learning experiences.
\end{abstract}

Indexing term/ Keywords:-Chinese; continuing professional education; longitudinal study; primary care physicians; qualitative study

Academic Discipline And Sub-Disciplines:-Medicine, primary care SUBJECT CLASSIFICATION:-Article

TYPE (METHOD/APPROACH) :-Individual interview, qualitativeresearch

\section{Council for Innovative Research}

Peer Review Research Publishing System

Journal: International Journal of Research in Education methodology

Vol. 7, No.1

ijremeditor@gmail.com

www.ijrem.com 


\section{INTRODUCTION}

Evidence-based research can advance the knowledge of medical educationand primary health care [1]and improve the quality of health care services [2].Among different study methodsin medical education andhealth careresearch, qualitative studies are commonly adopted nowadays [3, 4].

Cross-sectional studies which offer snapshots of the data of interest are frequently found in the literature. Longitudinal studies capture data on the continuing changes of the phenomena of interest through repeated observations or interviews on the same participants over a period of time. Recall bias from the participants in the studies can be minimized by repeated timely data collections $[5,6]$. The association of interacting factors can be established in the time course [5, 6].Longitudinal study is a comparatively more reliable means to capture the changes of the phenomena by data tracking over a period of time and to establish the suggestions of causes $[5,6]$.

Longitudinal design is commonly adopted in health care research studies to examine the changes of phenomenon of interest including in the field of primary care [7-9]. But, primary care physicians (PCPs) are reluctant to participate in research studies [1]. PCPsworking in private sectorprovide patients with extended consultation time to tailor their needs. Therefore, provision of long hours of service restricts their time for other activities, including research and continuing professional education (CPE). Longitudinal studies require the participants to spend more time on study. Therefore, it is even more challenging to recruit PCPs to participate in longitudinal studies [10-13]. Their commitment to finish longitudinal studies is a major study barrier.

In Hong Kong, the health care system comprises a heavily subsidized public sector and a self-financing private sector. The public sector provides the public with the majority of the tertiary, secondaryand rehabilitation services. Patients areonly chargeda low and fixed fee for each attendance including consultation, investigation and medication. For example, patients attending the Government's general out-patient clinics are only charged HK\$45 (US\$5.8) per attendance. Private PCPs are still able to satisfy $70 \%$ of the community's primary health care needs [14]. PCPs play important roles in promoting public health and offering early treatment to patients with chronic diseases [15]. Private PCPs have a significant contribution in these areas.

The Medical Council of Hong Kong (MCHK) is a statutory body to assure and promote the health care quality, and foster the ethical standard of medical doctors through registration and Continuing Medical Education (CME) programme. CME programme aims at maintaining and enhancing the practice standard of medical doctors by offering them learning activities. The quality of the learning activities was maintained by an accreditation system implemented by the MCHK. Medical doctors are registered as specialists or non-specialists with the MCHK. Specialists have completed formal specialty training, such as Family Medicine training while non-specialist doctors have not. Although the non-specialist doctors do not have any specialty training, most of them are practicing as PCPs. The MCHK encourages non-specialist doctors to participate in a voluntary CME programme through an incentive system to maintain and promote their practice standard. Knowledgeable PCPs are expected to alleviate the health care burden of a community [15].But, the non-specialist PCPs are not required to fulfill the requirements of mandatory CME programme on renewal of their annual registration with the MCHK. Their learning activities were not gauged and their learning barriers were not known as well. Hence, a thorough understanding of the situations with CME and CME non-accredited learning activities among them is essential for the health care policy makers and medical educators to design and implement health care policy and educational programmes. In this study, CPE referred 
to any practice relevant learning activities, including CME activities and CME non-accredited learning activities. The former ones included CME lectures, workshops and post-graduate study programmes offered by universities and Colleges of various specialties. The usual formats of the latter oneswere reading books and journal articles, on-line self-study, round table discussion with colleagues and consulting specialists on clinical problems. Challenges and coping strategies for conducting longitudinal qualitative studies on Chinese PCPs about medical education were not well explored. We successfully conducted a 12-month longitudinal qualitative study to explore the barriers and facilitators to undertaking CPE among Chineseprivate non-specialist PCPs.

No studies in the area of conducting longitudinal study on Chinese PCPs are identified. This article reporting the findings in these areas serves as a reference for researchers to work on Chinese PCPs in the future.

\section{METHODS}

\subsection{Background of the researchers}

In Hong Kong, two Chinese researchers conducted this study from $1^{\text {st }}$ Oct., 2011 to $30^{\text {th }}$ Sept., 2012. MKP is a private non-specialist PCP who has been providing primary health care in the community for 20 years. He is also a committee member of a CME organizer and regular members of some major medical organizations. TPL, a professor of Family Medicine and Primary Care, has over 15 years of experience in conducting qualitative research on medical educationandprimary health care.

\subsection{Allocation of participants into age interval groups}

We undertook this longitudinal qualitative study on the barriers and facilitators to undertaking CPE among the Chineseprivate non-specialist PCPs in Hong Kong. Data collections were taken in the forms of self-administered questionnaires and multiple individual interviews. We tracked and explored their learning experiences and viewpoints over 12 months. In this period, we investigated the effects of seasonal changes, community health careissues and significant yearly events of one year cycle on their learning.

Seven male and seven female Chinese private non-specialist PCPs aged of 25 or above in a wide demographic diversity were invited to participate in our study. The lower limit of age range was 25 because freshly registered medical doctors were usually at or above 25. Participants were assigned into age interval groups of 10 years each, resulting in five age interval groups. Participants in the fifth age interval group were 65 or older. The upper age limit for the participants was not specified as long as they were still practicing. One participant was allocated to each gender group. Two male and two female additional participants belonging to the third and fourth age interval groups were invited because a higher proportion of practicing private non-specialist PCPs was known to be within these age ranges.

\subsection{Data collection methods}

This study consisted of two data collection methods to complement each other. The first method was through inviting the participants to record all their CPE events in self-administered questionnaires. The participants were requested to return them to us every month. In the questionnaire, we defined an eligible CPE event as a practice relevant learning activity which lasted for 30 minutes or more irrespective of the learning format. We selected 30 minutes as the cut-off point to avoid overburdening the participants to fill in many questionnaires. Based on the findings of a pilot study, we finalized the 
questionnaire to 16 close-ended questions with multiple options for their answer choices. We aimed at further reducing their burden by designing the questionnaire in a way to allow the participants to finish them within one minute.

The second method of data collection was through conducting individual interviews. Each participant had three 20-minute four-monthly interview sessions and one 20-minute post-CME lecture interview session. All participants agreed on being audio recorded. We conducted one 10-minute post-interview discussion for all interview sessions with all participants. The post-interview discussion aimed at exploring the interview content and process. In the four-monthly interview, we focused on the personal, family, social and community health events which influenced their learning experiences in the preceding four months. In the post-CME lecture interview arrangement, we scheduled one participant and either of us to attend the same CME lecture. In the "natural" learning environment, the attending researcher, acting as an participatory observer, jotted down the field notes without any audio or video recording to avert the recording of the images and behaviors of other attending doctors in the event. Shortly afterward, the attending researcher conducted a 20-minute interview with the participant, focusing on his/her purposes of attending the lecture, and comments on the event process and content. It was followed by a 10-minutes post-interview discussion.

While we conducted the study, we obtained descriptive statistics from the returned questionnaires and performed thematic content analysis on the transcripts of the interviews and post-interview discussion notes [16]. The findings of questionnaire and thematic content analysis informed us the areas for further exploration in subsequent interviews.

We kept a research diaryrecording the conduction process, experiences and self-reflections on our study. We analyzed these qualitative data by thematic content analysis with theassistance of NVivo 9 (QSR International, Victoria, Australia) for writing up this article.

This study was approved by the Institutional Review Board of The University of Hong Kong/ Hospital Authority Hong Kong West Cluster (\#UW 11-300).

\section{RESULTS}

Thirteen participants completed the study and only one participant suspended her CPE in the second half of our study because of the management on her newly diagnosed acute myeloid leukemia.

\subsection{Challenges in recruiting participants}

Participants' commitment to finish our study was our major concern. Time allocation to different duties and tasks was an important barrier. Many self-employed private PCPs in solo practice had difficulties to spare their time to participate in our study because their incomes depended on the duration of their working hours. They provided patients with long hours of primary health care service to pay for their high office rent and business expenditures. Furthermore, in private sector, compensated hours were usually unavailable for PCPs to take part in research study. An invited participant reflected to us that he worked seven days a week. He squeezed all his time, even sacrificing his lunch time, to see as many patients as possible to pay for the high business expenditures. Despite his busy clinical work, he still participated in our study because he wanted his exhausting working conditions be known. Most PCPs were very busy with their practices. We encouraged them to join our study by emphasizing the recognition of the value of their time spent in this study and implicationsof the studyto our profession. 
Some PCPs, especially the younger ones, were busy establishing their own practices by extending their consultation hours and building their social and professional networks. Their main interests were usually undertaking CPE to enhance their knowledge and clinical competence, rather than taking part in research study to contribute to the advancement of medical knowledge. The older PCPs were more interested in their hobbies, social and family activities because financial burden was no longer an important issue to most of them. In spite of these challenges, we managed to invite PCPs to participate in our study by seeking the assistance from opinion leaders and keeping close liaison with local medical organizations whichhadclose relationship with PCPs.

PCPs worried about being asked sensitive questions. Some PCPs considered some personal, family and business related questions being private and sensitive. To address their concerns, we repeatedly reassured them that we would not ask them the questions on the business aspects of their practice and they could refuse to disclose any information on these areas. In addition, we emphasized that the identities of the participants would remain anonymous. The collected data were solely for the research purposes and would be kept confidential. To further uphold the appropriateness of the questions posed in interviews, we invited qualitative researchers of our department to advise us on the areas for further exploration in subsequent interviews. Their perspectives helped us have a deeper understanding on the general picture from the study findings.

Participants from a wide diversity of demographic background could enhance the richness of the data. Yet, it was a challenge of achieving this aim. Although snowball sampling is an efficient and commonly adopted recruitment method in interview studies, the referring medical doctors and their referred participants are usually relatively homogenous in demographic background. Consequently, recruitment through one or two referring doctors would limit the demographic diversity. This challenge was managed by seeking help from medical organizations and CME organizers. We could further enhance the success rate of recruitment with the endorsement from the opinion leaders of our profession,the significant committee and council members of these medical organizations. MKP had close working relationships with some private specialists who established their own patient referral PCP networks. Seeking help from them, we could further broaden the recruitment sources and enhance the heterogeneity of participants.

Besides widening the recruitment sources, trustful personal relationships with the participants and face-to-face invitation were the two other important factors for successful recruitment.Nevertheless, it was difficult to develop trustful personal relationships with potential candidateswithin a short period of time. Our established professional networks were imperative for us to reach and recruit the participants of a wide range of demographic characteristics. Endorsement from significant persons of our profession assisted us to recruit the PCPs to whom we were not familiar.

\subsection{Challenges in recruiting the less active learners}

To enhance the richness of data, we invited participants engaging in different levels of participation in CPE activities. The learning behavior and experiences of the active CPE learners might be different from that of the less active ones. Sampling of participants of "maximum variation" in learning activities could deepen our understanding on the subject. However, no official data gauging their CPE learning activities was available and we did not have the definition of less active learners. We could only check whether the PCPs were listed with active or inactive CME status in the database of the MCHK. This CME participation status checking procedure helped us to identify the PCPs with lesser CME learning activities only, rather than their levels of participation in CPE activities. Furthermore, we could not assume that the PCPs who seldom attended CME 
learning activities were less active CPE learners because they could still pursue CME non-accredited learning activities. It was also a challenge of identifying the PCPs who were less active in both CME and CME non-accredited activities because we did not have any information on their personal or social characteristics. The less active CPE learners were the hard-to-reach sub-population because of their social desirability consideration for disclosing their learning activities to us. These challenges could only be partially overcome by our close personal relationship with PCPs and multiple medical organizations.

Even though we were able to recruit some less active learners, we worried that they could not finish the whole study. A PCP who had never attended any CME activities in the past three years told us that she would not undertake any CME or CME non-accredited activities in the following 12 months. She confessed that even though she agreed to participate in our study, she could not promise us to finish the whole study.

\subsection{Challenges in enhancing the success rate of recruitment}

Most PCPs were trained in the traditional undergraduate medical curriculum which emphasized didactic teaching. They had little exposure to research studies and limited knowledge about research ethics. Even though we could identify the information rich participants, we took effort to convince them that their contributions to the advancement in medical knowledge were invaluable and their rights were well protected. It was important to explain to them about the relevance of our study to their practice, the research purposes, procedures and implications, incentive, data confidentiality and identity anonymity. By emphasizing that our study was university-affiliated, we could further convince them that our stance was neutral and the findings were used for academic purposes only.

\subsection{Challenges in organizing reminders and communication tools}

Repeated email reminders could improve the return rate of the questionnaires. Although telephone call reminder was commonly adopted in other studies, it was an unwise option in our study. Most of our participants disliked being disturbed during their office hours or intruded into their private personal lives. Hence, it was better to remind them through email reminders. We also took the opportunities to remind them when we met them in CME lectures. Sometimes, we passed the messages with the assistance from their referring doctors.

At the time of the study, instant messaging applications of smartphones were not widely adopted by the participants and some of them read emails only once every few days. One of the senior participants even did not have an email account. Consequently, communication between the participants and us was inefficient. On the busy working days, it was more difficult to contact them through emails or telephone calls.

In the latter part of the data collectionprocess, although the questionnaire consisted of only 16 questions, we recognized that repeatedly filling in questionnaires led to participants' response fatigue. We had to spend more effort in reminding them to fill in and return the questionnaires. Response fatigue was also a challengeof conducting interviews.

\subsection{Challenges in data collection in the four-monthly interview}

In addition to the collection of verbal information, data collection in the form of face-to-face interview allowed us to pick up non-verbal information to enrich our data. To facilitate the participants to finish the study, we let the participants decide the interview format, whether face-to-face or telephone interview, at their convenience. However, they were still unable to spare 
enough time for telephone interview because of their busy work. Sometimes, we could only conduct interviews in unsatisfactory conditions, such as face-to-face interview while participants were tutoring their children with their homework, and telephone interviews when participants were dining and walking. They were so busy that they might call us for telephone interviews when they were available. Thus, mobile telephone service provider with reliable signal network was crucial and our mobile phone had to be ready for recording all the time.

Most participants only agreed to have the conversations about their CPE experiences and opinions recorded. To soothe their worries about recording our private conversations, we arranged two telephone lines for the study. One was used for communication between the participants and us, and the second one was for telephone interview and recording. This arrangement helped them to feel safe to talk to us about some sensitive issues over the first telephone line. The post-interview discussion was also conducted on the first telephone line. On finishing this study, we concluded that this arrangement was effective toalleviatetheir worries.

\subsection{Challenges in arranging the post-CME lecture interview}

To arrange post-CME lecture interviews with the participants was even more challenging than the four-month interviews. Participation registration was commonly required for attending CME lectures. CME organizers prohibited non-members or walk-in doctors to participate in their lectures. To obtain the eligibility to attend the lectures together with the participants, we had to make requests to the CME organizers to grant us approval thatallowed the participants and us to attend the same lecture. On some occasions, either the participants or we, or neither party were the members of the CME organizers. These approval requests were only made possible if the CME organizers supported our study.

Because MKP was a committee member of a CME organizer, he was in a favorable position to arrange the participants to attend CME events. Yet, the locations of the participants' practices were scattered over the territory in Hong Kong where CME lectures were organized by different medical organizations. These organizations might hold different attitudes toward our study. Therefore, we had to spend more time in establishing the relationship with them.

Besides the challenge of granting approval, we faced the challengeof conducting post-CME lecture interviews in a face-to-face format at a specified time and place shortly after the lectures. This required the participants to sacrifice their time to participate in the scheduled event and interview amid their busy practice. Hence, we anticipated that we needed to spend more time in finishing all 14 post-CME lecture interviews. In total, it took us five months to finish all post-CME lecture interviews.

\subsection{Data of interest was also the barrier to conducting our study}

Data of interest could be the barrier to conducting our study. The incidental events, such as development of acute myeloid leukemia, death of father, domestic helper on leave and preparation for professional examination, not only affected the participants' learning experiences, but also brought them physical or psychological burden to continue participating in our study. Some participants simply did not reply to our email or telephone call reminders. To tackle the dropout problem, we recognized their difficulties by collecting their updated information through our professional networks. We took time and patience to convince them to continue our study and made flexible interview arrangements accordingly.

We knew little about the participants' personal information and had personal relationship with only a few of them. Although we established working relationships with them in the 12 months study period, we needed to keep them in their 
psychological comfort zone during interviews through tactful exploration of the areas of interest. Sometimes, we explored some areas which could enhance the richness of our data. However, we were not aware that the areas were too sensitive and should not be trespassed. The awareness of and prompt responses to their feelings, concerns, needs and worries helped us to build connection with them and avoid social embarrassment.

\subsection{Reflections from the participants}

At the end of our study, we sought reflections from the participants to evaluate the organization and process of our study. These could help researchers improve the design and implementation of longitudinal study among Chinese PCPs in the future.

Although the questionnaires were short and the total interview duration of an interview and a post-interview discussion was only 30 minutes, some participants got tired easily on repeatedly filling in the questionnaires and having multiple interviews. In private sector, the operation costs of their practices were so high that they needed to spend most of their time in consultation. At the end of the day, they were already very tired. To worsen the situation, their voluntary participation in our research study was an extra workload without any financial gain. Hence, some of them had ideas of dropping out from the study.

"I have not undertaken any longitudinal study as this one. It is so demanding. The workload is much more than I expected." $(\mathrm{S} 1-02)$

"I did not anticipate that I needed to spend so much time and effort in finishing this study. It was the first time and the last time. Please do not consider to invite me to participate in your future studies. "(S1-04)

"Because you are a committee member of my association, I agreed to help you. I organize and attend so many CME lectures that I am unable to fill in the questionnaires about all of them. Your reminders of filling in the questionnaires are the pressure that you put on me. Please do not remind me to return the questionnaires anymore." (S1-14)

Although the study burden on the participants was heavyand they were allowed to withdraw from our study without the need of giving any reasons, all of them kept their commitments to finish the study. Yet, they still needed their difficulties to be understood by us. After much discussion about the purposes and implications of our study, we managed to convince them to continue their participation. Some participants reflected that it was a difficult decision in choosing to keep their commitments or to drop out of our study. Some participants commented that they were relieved when they finished our study.

"Done. Bye bye. Today is my free day. " (S1-01)

“Done. Get released!" (S1-13; S1-11)

After they made much effort to finish our study, they felt contented that they helped us and contributed their information to enrich the knowledge about CPE.

"Although I am very busy, this study is relevant to our profession. I need to keep my promise to finish the study.I am proud of making advances in the understanding on medical education. " (S1-02)

Minimizing the inconvenience to our participants was on the top priority for our research design. We decreased the study 
burden by reducing the number of questions to 16 in the questionnaire and defining a CPE event lasting only 30 minutes or more. After they were familiar with the questionnaire, they took less than one minute to finish one. However, it may be unwise to shorten the duration of interviews because it was just sufficient to collect in-depth data within 20 minutes from the participants. If we had reduced the frequency of interviews, recall bias of the learning activities in the preceding months might have been a concern and our rapport with participants would be weakened as well. By shortening the interview duration, we will sacrifice the richness of the collected data and have more difficulties to understand their worlds.

Some participants told us that their significant friends strongly recommended them to join our study. Their reflections supported our conclusion that our professional networks and practice relevance of our study were the major facilitators of completing our study. Seeking help from their respected colleagues was an effective way to recruit and encourage the participants to finish our study. Close personal relationship with the significant persons in our profession helped us to solve many challenges in this study, such as exploringthe reasons of refusals from participants

"It was endorsed by my respected senior doctor and mentor. Otherwise, I would not agree to help you. Once agreed, it becomes my duty! Sometimes, he asked me how I was doing with the study. How could I withdraw from your study?" (S1-09)

We had difficulty in differentiating sensitive questions from insensitive ones because we did not know the participants' background in detail beforehand. In telephone interviews, without the cue of the participants' body language and facial expressions, we had no idea of the participants' attitudes toward our questions. For example, one participant mentioned that she did not expect uncomfortable questions posed in the telephone interview.

"Some questions ... are too private and sensitive. I do not predict that you will ask these questions beforehand. For example, I do not want to compare the quality of CME lectures that were organized by various CME organizers." (S1-09)

We increased the heterogeneity of the participants by widening their demographic background diversity. Therefore, their response attitudes to the same questions were different. On further probing the field of interest in interview, we needed to remain vigilant all the time about their emotional responses and body language.

The positions in the hierarchy of social status between participants and us were similar. Neither party dominated the interviews. This relationship favored us to guide the interview in the direction of exploration. Conducting interviews with the assistance from research assistant was not a good idea. PCPs considered that research assistants were outsiders of the medical profession and should not attend the interviews.

Although all participants commented on the negative aspect of our study, two senior participants reflected that they enjoyed the study process as a form of social activity and learning. By helping the participants to enjoy the participation process, we could capture in-depth dataon the subject by widening their psychological comfort zone in interviews.

"Meeting you in the interview is a social event. It is very boring for me working all day in my clinic. I am happy to meet you again today." (S1-06)

"I have learned a lot from you and have made friend with you. Making friend with you is my pleasure." (S1-07)

\section{DISCUSSION}

Our professional networks and the medical professional status facilitated us to recruit participants and conduct our study. 
Inactive learning is socially undesirable among medical doctors. Identification of less active learnerswas a challenge because this group of PCPs was well 'hidden' even to their colleagues. They also readily declined participation invitations of studies about medical education. Further research on identifying and recruiting this group of PCPs are required for future studies. The time demand of longitudinal study on participants is heavy. In recruitment, it is wise to let participants understand the impact of participation on their work, family and personal life. Some PCPs still found that participation in any research studies was irrelevant to them. It is probably due to their limited exposure to research studies. Explaining the significance and process of research studies to this group of PCPs will encourage them to particpate in future studies.

Both the participants and we arePCPs. It was easier for us to recruit participants, build rapport and work in collaboration with them. Our profession helped us have some prior understanding of the subject for exploration. However, our theoretical sensitivity to develop the theory of the subject might be weakened by focusing on our own experiences and overseas study findings. Theoretical sensitivity is the personal quality of researchers. Grounded on data, researchers are able to be aware of the subtleties of data, gain new insight and develop new theory on a subject $[17,18]$. During interviews, we played three roles in the interaction with the participants. The first role was an interviewer, the second a colleague and the third a friend. These roles strengthened our theoretical sensitivity by viewing and analyzing the whole study in multiple lenses[18]. However, we always needed to keep our mind open, be aware of our assumptions and develop our theory grounded on data $[17,19]$. The challenge of theoretical sensitivity was managed by our reflexivity through repeated self-reflection, maintaining skepticism on the hypotheses, categories of data and explanations of phenomenon [19]. We also invited experienced qualitative researchers to comment on the conduction process, interview questions and data analysis from their perspectives. Therefore, we were able to avoid forcing the existing theories and our own experiences into our understanding of the subject [20].

\section{CONCLUSIONS}

All participants accepted our invitation mainly based on the relevance of our study to their practice and our profession status [12].Clear explanation of the purposes, implications of our study and their rights in research study helped them know what they were expected. Longitudinal qualitative study is very demanding on participants. It is better to avoid overburdening them. Empathy, concerns and care are essential elements to build up rapport with our participants. With appropriate planning, strategies and patience, it is feasible to finish a longitudinal study on Chinese PCPs.

\section{ACKNOWLEDGEMENTS}

The authors wish to thank the assistance of Dr. Eric WC Tang, Dr. Aaron FK Lee, Dr. YK Law, Dr Joseph SY Lam, Dr. WK Chau and Dr. PY Yik to recruit the participants and endorsed the study. All of them offered invaluable support in the whole process of the study to help the researchers to complete the work uneventfully.

\section{REFERENCES}

[1] Asch, S. C.,Sarah, E. B. A., Hamilton, E. G. M. S.et al. 2000.Problems in recruiting community-based physicians for health services research. J. Gen. Int. Med. vol. 15,no. 8, 591-599.

[2] Lam,C. L. K.2004. The 21st Century: The Age of Family Medicine Research? An. Fam. Med.vol. 2,Suppl. 2, S50-S54,http://dx.doi: 10.1370/afm.191. 


\section{ISSN 2278-7690}

[3] Bradley, E. H.,Curry, L. A.,andDevers, K. J.2007. Qualitative data analysis for health services research: developing taxonomy, themes, and theory.Health Serv.Res., vol. 42, no. 4.1758-1772.

[4] Green, J.,and Britten,N.1998. Qualitative research and evidence based medicine.BMJ. vol.316, no. 7139, 1230.

[5] Bernard, H. R. 2006Anthropology and the Social Sciences Research Methods.In Research Methods in Anthropology: Qualitative and Quantitative Approaches.1-27, Alta Mira Press.

[6] Pope, C.,and Mays, N. 2006 Qualitative methods in health research. In Qualitative research in health care. 1-11, Blackwell Publishing Ltd.

[7] Booth, B. J., Zwar,N.,and Harris,M. F. 2013. Healthcare improvement as planned system change or complex responsive processes? A longitudinal case study in general practice. BMC Fam. Pract. vol. 14, 51. http://dx.doi:10.1186/1471-2296-14-51.

[8] Haggerty, J., Fortin, M.,Beaulieu, M. et al.2010. At the interface of community and healthcare systems: a longitudinal cohort study on evolving health and the impact of primary healthcare from the patient's perspective. BMC Health Serv. Res.vol. 10, 258-258.

[9] Senneseth,M., Alsaker, K.,and Natvig, G. K. 2012. Health-related quality of life and post-traumatic stress disorder symptoms in accident and emergency attenders suffering from psychosocial crises: a longitudinal study. J. Adv. Nurs.vol. 68, no. 2, 402-413.

[10] Green, J. S.,and Kristofco, R. E. 2011 The Meaning and Value of Continuing Medical Education in Continuing Medical Education: Looking Back, Planning Ahead . 359-368, University Press of New England.

[11] Cave, A.,Ahmad,E.,andMakarowsk, C. 2009. Recruiting issues in community-based studies: some advice from lessons learned. Can. Fam.Physi.vol. 55, no. 5,557-558.

[12] Johnston, S., Liddy, C., Hogg, W.et al. 2010.Barriers and facilitators to recruitment of physicians and practices for primary health care health services research at one centre. BMC Health Serv. Res.vol. 10, no. 1, 109-116.

[13] Shelton, B. J.,Wofford,J. L.,Gosselink, C. A.et al. 2002. Recruitment and retention of physicians for primary health care research.J. Comm. Health.vol. 27,no. 2, 79-89.

[14] Bureau of Food and Health. 2008 Your Health Your Life-Healthcare Reform Consultation Document. Hong Kong.

[15] Starfield, B., Shi, L., and Macinko, J. 2005. Contribution of Primary health care to Health Systems and Health. Milbank Quarterly, vol. 83, no.3, 457-502.

[16]Richards, L. 2009. Setting up your project. Handling Qualitative Data: A Practical Guide.11-32, SAGE Publications Ltd.

[17] Hoare,K. J., Mills, J., and Francis,K. 2012. Dancing with data: An example of acquiring theoretical sensitivity in a grounded theory study. Int. J. Nurs. Pract. vol.18, no. 3, 240-245.http://dx.doi: 10.1111/j.1440-172X.2012.02038.x

[18] Orland-Barak, L. 2002. The theoretical sensitivity of the researcher: Reflections on a complex construct.Refl.Pract.vol. 3, no. 3, 263-278.

[19] Thornberg, R. 2012. Informed Grounded Theory," Scand.J. Educ. Res.vol. 56, no. 3, 243-259.http://dx.doi: 
10.1080/00313831.2011.581686.

[20] Blythe,S.,Wikles, L., Jackson, D. et al. 2013.The challenges of being an insider in storytelling research.Nurs. Res.vol. 21, no. 1,8-13. 